\title{
Merlin: A Language Server for OCaml (Experience Report) ${ }^{\star}$
}

\author{
FRÉDÉRIC BOUR, Facebook, France \\ THOMAS REFIS, Jane Street, UK \\ GABRIEL SCHERER, INRIA, France
}

We report on the experience of developing Merlin, a language server for the OCaml programming language in development since 2013. Merlin is a daemon that connects to your favourite text editor and provides services that require a fine-grained understanding of the programming language syntax and static semantics: instant feedback on warnings and errors, autocompletion, "type of the code under the cursor", "go to definition", etc.

Language servers need to handle incomplete and partially-incorrect programs, and try to be incremental to minimize recomputation after small editing actions. Merlin was built by carefully adapting the existing tools (the OCamllex lexer and Menhir parser generators) to better support incrementality, incompleteness and error handling. These extensions are elegant and general, as demonstrated by the interesting, unplanned uses that the OCaml community found for them. They could be adapted to other frontends - in any language.

Besides incrementality, we discuss the way Merlin communicates with editors, describe the design decisions that went into some demanding features and report on some of the non-apparent difficulties in building good editor support, emerging from expressive programming languages or frustrating tooling ecosystems.

We expect this experience report to be of interest to authors of interactive language tooling for any programming language; many design choices may be reused, and some hard-won lessons can serve as warnings.

CCS Concepts: • Software and its engineering $\rightarrow$ Integrated and visual development environments; Incremental compilers; • Theory of computation $\rightarrow$ Parsing; Functional constructs;

Additional Key Words and Phrases: Development environments,language servers,incrementality,incremental parsing,incremental typing,parsing,syntax error recovery

ACM Reference Format:

Frédéric Bour, Thomas Refis, and Gabriel Scherer. 2018. Merlin: A Language Server for OCaml (Experience Report). Proc. ACM Program. Lang. 2, ICFP, Article 103 (September 2018), 15 pages. https://doi.org/10.1145/3236798

\section{INTRODUCTION}

The research communities that study programming languages have demonstrated their ability to propose interesting, valuable new programming constructs and whole languages. Yet a major difficulty to disseminate those ideas is the scarcity of tooling for those research languages. We have built conceptual and practical tools that let us design and implement interesting languages in a few man-years, but building a satisfying tooling ecosystem remains much more work-demanding, and seems reserved to industrially-backed languages.

The present report is concerned with the tooling support traditionally provided by IDEs (Integrated Development Environments), namely language-aware editor features, letting programmers interact with the source of a program as they are writing, modifying or inspecting it. Proponents

${ }^{*}$ Due to space limitations, substantial portions of the article were omitted from this short version. We encourage the reader to instead consult the full article (24 pages) at https://arxiv.org/pdf/1807.06702.

This work is licensed under a Creative Commons Attribution-ShareAlike 4.0 International License.

(C) 2018 Copyright held by the owner/author(s).

2475-1421/2018/9-ART103

https://doi.org/10.1145/3236798

Proc. ACM Program. Lang., Vol. 2, No. ICFP, Article 103. Publication date: September 2018. 
of a new or niche programming language wish to provide language support for all major IDEs and editors, and the only practical way to do this is to build tools to share the language-awareness logic among many different editors. We use the term language server to refer to a tool providing this language-aware logic ${ }^{1}$. Merlin is a language server for the OCaml programming language.

From the user point of view, language servers provide instant language-aware feedback in response to editing actions, with the editor signaling syntax errors, typing errors and warnings, or making auto-completion suggestions. They also enable various explicit user actions, interrogating the partial program (what is the type of this identifier?), navigating the project (go to definition, go to enclosing function...), or performing editing actions (complete this identifier, generate a covering pattern-matching on this expression...). Adding support for a new editor to a language server requires no language-specific logic: the editor plugin is merely a shim that continuously sends the partial program to the server, and exposes user-interface actions corresponding to the language-aware server features. Merlin currently provides, through a command line interface, language-aware editing modes for Vim, Emacs, Sublime Text, Acme, Atom, VSCode and Intelli-J.

Let us take just one simple example, given the following, still incomplete, piece of code:

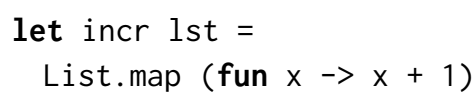

the programmer can place their editor cursor on any $x$ of the second line and hit a key to get its type. Under the hood, the editor then invokes a command looking something like this:

ocamlmerlin server type-enclosing

-position 2:16 -index 0 -filename test.ml -verbosity 0

passing the content of the buffer ${ }^{2}$ on stdin. This command returns the following JSON object on standard output, to be interpreted and rendered by the editor appropriately

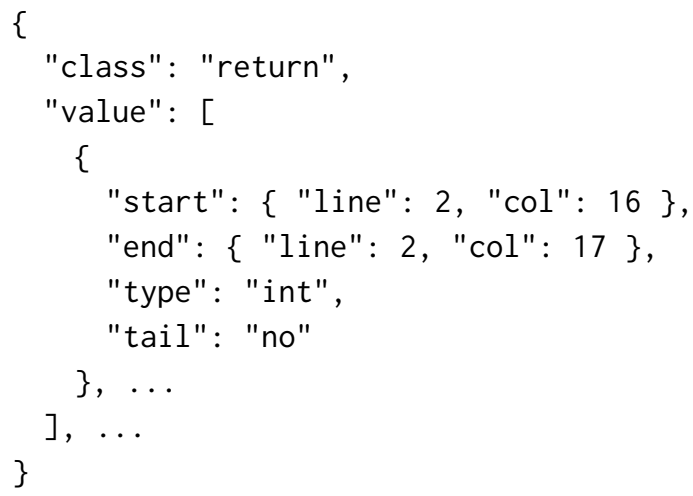

For the experience to be smooth for the programmer, merlin, as indeed any other language server, is expected to answer such queries in a few hundred milliseconds, even for projects with hundreds of modules and buffers of several thousand of lines. Besides type-enclosing, other examples of commands supported by merlin are complete-prefix for completion, locate to find the definition of a name and errors to display warnings and errors.

\footnotetext{
${ }^{1}$ The terminology comes from Microsoft's Visual Studio Code editor, which introduced a standard communication protocol between editors and language servers called the Language Server Protocol. Merlin (2013) was created before Visual Studio Code (2015), and does not use this specific protocol - for now.

${ }^{2} \mathrm{~A}$ "buffer" is a single piece of text or source code that is currently being edited in a text editor. Buffers usually correspond to files on the filesystem, but they may have changed since the last "save" operation or not have been written to disk at all.
} 
The main technical piece required to build a language server is a language frontend (as found in typical language implementations: parsing and static analysis) that is reasonably incremental (for responsiveness) and supports partial programs with missing or erroneous program fragments. The language server continuously receives the current partial program, and computes a partial typedtree - a typing derivation for the partial program. Language-aware queries are then implemented by processing this partial typed-tree.

One specificity of Merlin is that it was not built by implementing a new language frontend, but by modifying existing tools to build non-incremental frontends (for any language) to support incrementality and partiality. These general extensions enabled many more uses than just a language server, and we will report on some of them.

Not everything in a language server is principled; Merlin makes some simplifying assumptions that have proven robust in practice, but also found out that some things are more difficult or fragile than they first seem. We hope that this experience may help other authors of language servers.

We claim the following contributions:

- A presentation of how to adapt the interface of lexer and parser generators of the lex/yacc family to support incrementality and partiality, a discussion of use-cases, and a (less general) discussion of incremental/partial type-checking.

- An exposition of a language server design and implementation, both principles and heuristics, which could be of interest to implementors of language servers. We present the ideas in Merlin in a language-agnostic way when possible, and point out when specific language feature or ecosystem characteristics created unexpected difficulties.

Article Structure. We chose to focus this short version of our article on the general design structure of Merlin (Section 2) and the important differences compared to a traditional compiler frontend (Section 3), namely incrementality and partiality.

In the full version of this article (https://arxiv.org/pdf/1807.06702), we also discuss the other adaptations to the frontend, describe a few language-aware features of interest, detail a few of the difficulties or challenges encountered during the development of Merlin, and finally provide some thoughts on the relations between language tooling and language design.

\section{THE MERLIN MODEL}

In this section we will present the successive interaction models Merlin has exposed since it was first written, these evolved over time in response to implementation constraints and experience.

It gives a high-level perspective of how Merlin interacts with the editor, and of the information they exchange.

\subsection{A Reminder on the OCaml Compilation Model}

OCaml source files may be either implementation files (foo.ml) or interface files (bar.mli); an implementation file contains the source of an ML module, and an interface file contains its signature. An implementation and interface file of the same name, foo.ml and foo.mli, together form a compilation unit foo, which is visible in the language as a ML module Foo (the same name, capitalized).

When source files for a compilation unit foo are passed to the compiler, it produces some compiled implementation files, and a compiled interface file foo. $\mathrm{cmi}$; if the compiler is given a file which uses the module Foo, it only needs to access foo.cmi to type-check this use. This separation of implementation and interface enables incremental and separate compilation.

Compiling the source of a compilation unit requires knowing about the other units that it depends on. The compiler runs in a given compilation environment, which is a mapping from module names 
to compiled units on the filesystem. (In particular, this compilation environment determines the initial typing environment to type-check the current compilation unit.) This mapping is built from the already-compiled files in the current project and from the installed third-party libraries whose path was passed to the compiler at invocation time.

OCaml enforces an acyclic dependency graph between compilation units: a program or library is an ordered list of units, each depending only on units before it - and, in the case of libraries, on third-party units to be linked separately.

\subsection{The Old Build and the Buffer}

When the user is editing a source file buffer with a Merlin-enabled editor, Merlin will continuously receive the partial buffer state from the editor. Just like the compiler, it reconstructs the compilation environment from compiled interface files, found either in the current project (typically coming from an old build of the project) or from third-party libraries.

From the point of view of Merlin, there is thus a single buffer being edited, which requires special incrementality and partiality support, and a set of compiled interface files that are used in the standard way to build a typing environment.

Merlin supports editing several file buffers at once, but each buffer belongs to an independent session, with no cross-session communication. Merlin never tries to synchronize or share partial information from several files being edited simultaneously. In practice this heuristic simplification works very well, for at least two reasons. First, each session typically has access to a mostly-correct interface for the other files coming from an old build. Second, programmers typically spend time editing implementation files foo. $\mathrm{ml}$ without breaking the interface file foo.mli, allowing new builds to create correct a foo. cmi even if the whole build fails on partial files.

\subsection{Evolving the Interaction Models}

2.3.1 The Toplevel Model and Linearization. The first interaction model used by Merlin was inspired by interactive toplevels (read-eval-print loops). Merlin would interpret sentences of the buffer in order, up to the first point with a hole or an error. Early version would even show a "blue zone", inspired by the Proof General interface, to provide visual feedback on which prefix of the buffer had been type-checked. A first prototype of such an approach is easy to build on top of a stock parser found in an interactive toplevel - the OCaml compiler distribution comes with such a toplevel, who shares its grammar description with the compiler. This model also offers some weak error-resilience (partiality support) for cheap: phrases containing an error can simply be skipped.

The main difficulty with this model is that the granularity of "toplevel phrases" used by toplevels is much too coarse to support partial programs in practice. In ML languages with a hierarchical module system, a phrase may be a complete module declaration with several sub-phrases. Consider the following example of partial program (at least an end terminator for struct . . end is missing):

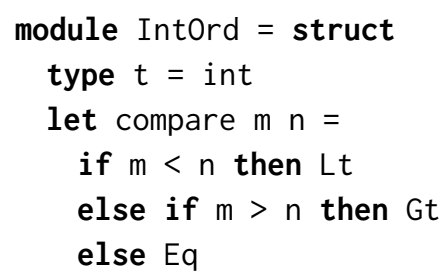

This incomplete module declaration is an incomplete/invalid toplevel phrase, but a user would still expect the tool to process the declarations of $t$ and compare. 
To satisfy this need, Merlin moved to a "linearized" grammar for OCaml, where nesting structures are turned into independent sentences allowing partial processing. A single-phrase module declaration (here we use the ugly optional OCaml phrase terminator ; ; for clarity)

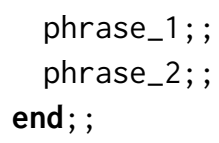

is represented, after linearization, by a series of phrases

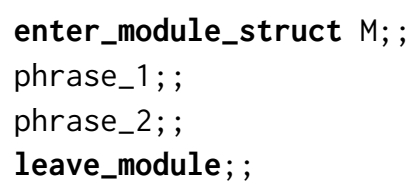

where enter_module_struct and leave_module are new Merlin-specific phrase-forming constructs that linearize the module $M=$ struct $\ldots$ end nesting - this is similar to the transformation of a tree grammar into a streaming interface. Note that this need for linearization is not specific to nested ML modules, it naturally arises with all nesting structures such as local declarations or list or record literals.

(This form of linearization is also present in Proof General interfaces, as we often want to have the "blue/checked zone" end in the middle of a nesting construct. The Proof and Qed/Defined commands of Coq are precisely linearizations of a document nesting structure.)

The Merlin authors modified the upstream toplevel grammar to parse this linearized grammar. However, they quickly found out that the effort to linearize nesting constructs was tedious, incomplete, and consuming more and more time. Not only must the parser be modified, invasive changes were also required in the type-checker to deal with these new constructs, with a different approach required for different kind of linearizations. The development and maintenance burden was much too important, and a new approach was necessary.

2.3.2 The Partial Compiler Model. The next iteration of incrementality support for Merlin used a standard LR grammar description for the OCaml grammar, with a parser generator modified to generate an incremental parser - we describe this design in Section 3.1.

This changed the interaction model of Merlin from acting as an (type-checking-only) interactive toplevel to acting like a (type-checking-only) compiler: the editor passes source buffers to Merlin, along with information on the compilation environment, and Merlin does its best to produce partial typing information from a partial Abstract Syntax Tree (AST) - stopping at the point where the incomplete buffer ends, where the first error was encountered, or where the user cursor was editing, whichever comes first.

Note that it does not matter, conceptually, whether such a "partial compiler" is fed incremental or non-incremental output from the editor: the whole pipeline is incremental and can recompute a result from previous states and a source change, but this source change may either be communicated by the editor itself or computed by Merlin from the entire new buffer (copying source text in memory is not a performance bottleneck) and previous states. ${ }^{3}$

The first iteration of this "partial compiler" represented partial programs with parser states, which essentially corresponds to an AST spine with the right-most nodes missing. Merlin implemented a

\footnotetext{
${ }^{3}$ How Merlin records previous states is an implementation detail. Merlin works as a daemon, so it keeps a cache in memory, but a short-running tool could persist state on the disk. The important property to ensure is that the presence or absence of saved state does not modify Merlin's results, only its performance. In fact, Merlin does provide a short-running mode that does not use any cache, which is slower but helps testing correctness.
} 
variant of the type-checker that works on these partial ASTs, rather than complete ASTs as in a compiler - we detail this in the long version of this article. This requires a bit of work for each AST construction, but adapting the type-checker code was surprisingly easy.

On the other hand, the logic to traverse the parser state and expose the partial AST to the typechecker proved cumbersome to synchronize with changes in the continuously-evolving OCaml compiler fronted. This approach would be a sensible choice if this piece of infrastructure was included in the upstream language implementation, but Merlin needed a more pragmatic approach.

2.3.3 The Completed Compiler Model. Merlin's technology for incremental parsing stabilized relatively quickly; an aspect of the parsing stack that improved gradually over time and keeps evolving today is error recovery, and in particular completion, which consists in completing the valid prefix of a buffer into a more complete program, either by just inventing new tokens or non-terminals to close off nesting structures - when reaching the end of the buffer - or by also trying to skip some parts of the buffer and reconnect later, after the error error or hole. We detail completion mechanisms in Section 3.2.

Once syntax completion got good enough to work reliably in practice, it became possible to abstract away the partial nature of the input AST: a partial syntax tree is completed by recovery. Instead of having the type-checker work on partial ASTs, it is thus possible to reuse a normal type-checker expecting ASTs. Adaptations to the type-checker are still necessary for error-resilience (some parts of the parsed syntax may not type-check, and the completed parts of the syntax may also break type-checking locally), but the input format is the same as for the standard compiler, and this facilitates code reuse.

2.3.4 Memoization in Absence of Incrementality. Recent versions of Merlin have tried to improve support for pre-processing mechanisms used in the OCaml community - the ppx syntax extensions. Those extensions rely on running external programs to transform syntax trees between the parsing and the type-checking phase; the API (Application Programming Interface) of these AST-rewriters is still evolving as tooling changes, but they currently do not provide an incremental interface. A preprocessor is fed the entire AST to transform, and may generate different code at the beginning of the tree depending on what happens later in the code, so the entire transformation result must be recomputed on each source change.

Discussion is ongoing with designers of syntax preprocessing libraries to enable incrementality, by letting extensions produce a serialization of their internal state after processing a part of the buffer, and advertise that their transformation commutes with addition of further phrases of input.

In the meantime, Merlin behaves in a non-incremental way on files which use syntactic preprocessing, using memoization to avoid as much recomputation as possible during the type-checking phase. Merlin considers the post-processed source a sequence of toplevel phrases, and saves the typing environment for each prefix sequence of phrases. After a buffer change, preprocessors may perform arbitrary change to the syntax tree, but if the result is close to the previous postprocessed output Merlin will only re-type-check the phrase currently being edited.

\subsection{Remark: Purity Prevails Again}

One notable design and implementation consequence of the move from a toplevel-like model to a compiler-like model is purity.

In the toplevel model, phrases are naturally understood as stateful actions on the typing state linearization turns declarative nesting structures into more stateful commands. Each Merlin session would keep the "toplevel state" of the buffer on its end, and various bugs showed up where the synchronization between editor state and Merlin state would break. Typically, knowing what to replay after a Merlin crash would require delicate and buggy communication with the editor.

Proc. ACM Program. Lang., Vol. 2, No. ICFP, Article 103. Publication date: September 2018. 
In the compiler models, the interface between the editor mode and Merlin is much simpler: the editor sends the text buffer to Merlin, as one would invoke the compiler on the file. Merlin's behavior is a pure function of its input; an internal cache is used to enable incrementality, but its presence only affects reactivity, not the output content, so a desynchronization at worst results in a slowdown.

We strongly recommend that language servers follow this pure model. The improvements in consistency for users and debuggability and maintenance for developers are considerable. This interface even allows hot-reloading a new Merlin version during an editing session, which proved very useful to develop Merlin itself.

\subsection{The Language and the Model}

\begin{tabular}{|l|c|c|}
\hline & definition before use & forward references \\
\hline definitions at toplevel & easy, linearized prefixes & $\begin{array}{c}\text { medium, full linearized } \\
\text { (Haskell, Erlang, Java) }\end{array}$ \\
\hline rich scoping structures & $\begin{array}{c}\text { medium, fine-grained prefixes } \\
\text { (ML, Lisp) }\end{array}$ & $\begin{array}{c}\text { hard, full fine-grained } \\
\text { (Scala) }\end{array}$ \\
\hline
\end{tabular}

Fig. 1. Language design aspects and incrementality model

In all these interaction models that Merlin went through, Merlin works best on the prefix of the buffer before the first error or incompleteness. Completion and recovery strategies help analyzing further than the first error, but there are always difficult cases where this situation is degraded. Furthermore, checking only up to the cursor, the place where operations and queries happen, is a robust simplification to speed up processing.

The fact that this strategy works well in practice relies on a particularity of the ML-family programming language: it is relatively rare for identifiers to be used before their declaration. ML languages do contain mutually-recursive declarations that allow forms of forward references, but user programs tend to make a moderate, local use of them.

In contrast, many languages, typically Haskell, Erlang or some object-oriented languages, rely on liberal uses of recursion nests; the model of working only with definitions above in the buffer does not lead to an acceptable user experience. On the other hand, linearization works much better in these languages, that have a simple top-level structure with less nesting of module/scoping constructs - one may linearize the whole buffer, dropping incomplete/erroneous phrases. Languages with both rich scoping structures and abundant forward references, like Scala, would be harder to cope with. We summarize this design space in Figure 1.

\section{CHANGING THE FRONTEND}

The Merlin authors explicitly tried not to reimplement a new OCaml frontend, but to reuse existing tools and adapt them to support incrementality - and recovery. The specific tools used are implemented in OCaml and used within the OCaml community, but they follow a common heritage of lex-inspired lexer generators and yacc-inspired parser generators - and an extended implementation of the Hindley-Milner type inference algorithm. There are similar tools within most language communities.

\subsection{Incremental Parsing: Adapting Menhir}

The first toplevel-like model for Merlin (see Section 2.3.1) used a very simple incremental parsing strategy: it would parse the buffer phrase by phrase - using existing support in the OCaml grammar 
for toplevel phrases - and catch the exceptions raised on reaching end-of-input. After an editing action, only the edited phrase needed to be reparsed. When Merlin moved to a compiler-like approach (Section 2.3.2), a new approach to incremental parsing was required.

The OCaml compiler uses the OCamlyacc parser generator, inspired from its C counterpart Yacc. It takes a grammar description in Yacc style (including a list of tokens), and generates a parser for each declared start symbol.

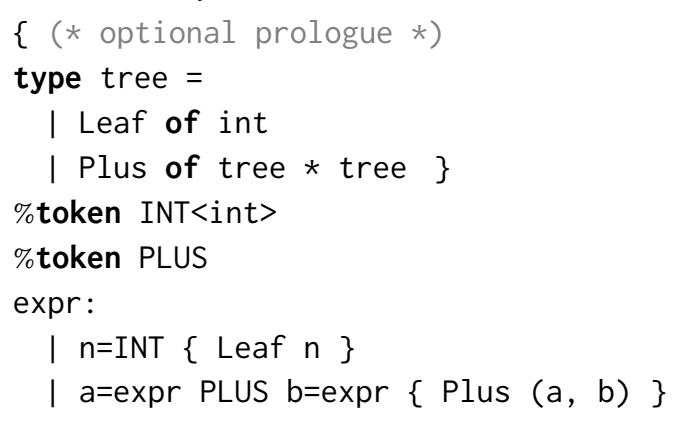

The generated parser for this grammar will have the following interface:

exception Failure of Lexing.position

val expr: (Lexing.lexbuf $\rightarrow$ token) $\rightarrow$ Lexing. lexbuf $\rightarrow$ tree

The generated parser takes a (lexbuf-consuming) lexer and produces a lexbuf-consuming parser. This parser will repeatedly invoke the lexer on the input buffer, producing a complete AST tree out of the resulting token stream - or raise an exception on parse error.

As for lexers ( see the long version of this paper ), the generated function assumes full control over the parsing process ${ }^{4}$, and we need to give control back to the user to bring support for incrementality and partiality. The Merlin authors developed an incremental parsing interface for Menhir, a parsing generator that is compatible with OCamlyacc's grammars; it is implemented in OCaml rather than $\mathrm{C}$, so more convenient to develop and extend. They proposed a new "incremental mode" for Menhir, that generates a different interface: ${ }^{5}$

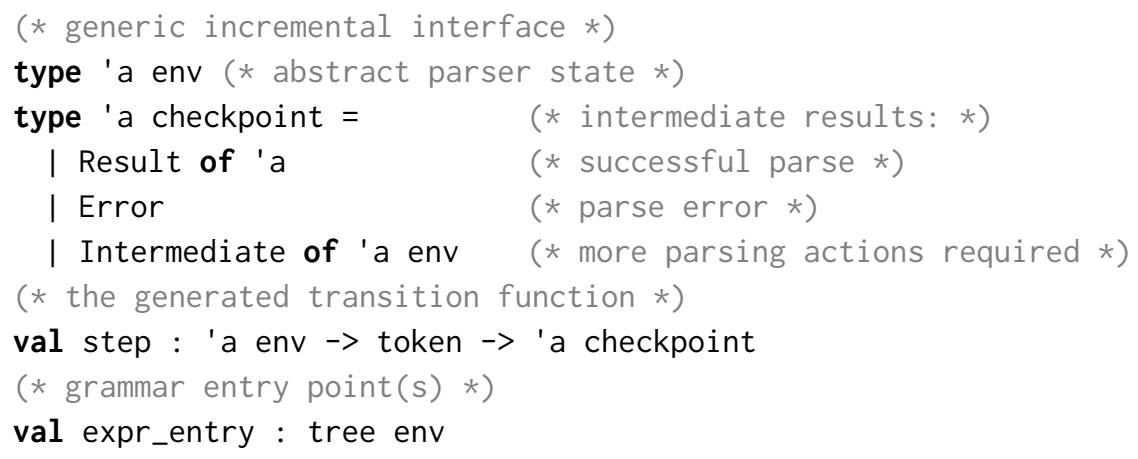

Most Yacc-style parser generators, Menhir included, use a parsing strategy in the LR family (LR, LALR, etc.). They generate a parsing automaton (a deterministic pushdown automaton). The parsing state is represented by a state number and a parsing stack (the innermost construction

\footnotetext{
${ }^{4}$ The in-control interfaces for lexing and parsing generators come from the original tools for $\mathrm{C}$ - where the lack of algebraic datatype makes finer-grained control cumbersome - and also from performance concerns: giving full control to the generated consumer lets it easily use local mutable state without paying serialization costs.

${ }^{5}$ We simplified the interface for the presentation: there are several Intermediate states, see the Menhir manual (Section 9.2, Incremental API).
} 
being parsed is at the top). Transitions (the dreaded shift and reduce transitions) go from one state to another, some requiring to consume a token from the input. In this incremental interface, ' a env is an abstract type for a parsing state that will eventually return a semantic value of type ' a or fail with an error, and the step function consumes a token and performs all transitions until a new token is required.

With this incremental interface, the user is in control of the parsing, feeding tokens one after another. The original interface is easily expressible as a parsing loop:

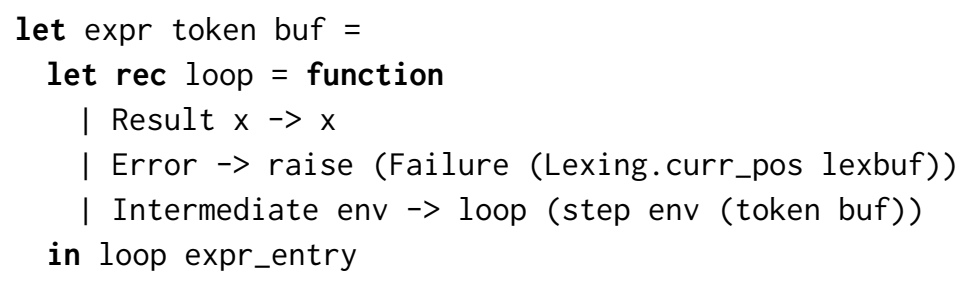

States and checkpoints are purely functional: they can be stored and reused at will. In particular, we get incrementality by caching them, indexed by token location: when a part of the buffer is modified, we can restart from the last checkpoint before it. There is a lot of sharing within parsing stacks and the semantic values on the stack, keeping the memory usage close to non-incremental parsing. We are assuming, of course, that semantic actions - the user-provided code running to build AST values - are pure as well, as they should be.

3.1.1 Inspecting the Parsing Stack. With the new interface, the state of the parser is available as a value. Merlin authors developed an advanced API to inspect (and modify) this value outside of the normal parsing process.

Giving access to the state of the parsing automaton, which is represented as an integer, is easy, but giving type-safe access to the semantic values on the parsing stack requires care. Each element of the parsing stack corresponds to a symbol (a terminal or non-terminal symbol), and contains the corresponding semantic value:

- for terminals, it is an arbitrary value passed by the lexer to the parser (e.g. when parsing an integer, the INT token will be generated together with the value that was parsed)

- for non-terminals, it is the value returned by the semantic action of a production rule (for this non-terminal) that matched the elements already present on the stack.

In particular, semantic values of different stack items may be at different types - in our example, int and tree.

Remark: This problem was discussed in detail precisely in the original article presenting Menhir, Pottier and Régis-Gianas [2006], which remarked that GADTs (Generalized Algebraic Datatypes) could be used to give a fully-typed presentation of an LR parser stack. At the time, OCaml did not have GADTs - they became available in OCaml 4.00, released in 2012 - so Menhir internally used unsafe type casts.

The Merlin authors extended Menhir to generate a stack-inspection interface using GADTs to type-check semantic values in a precise way. For our example, the following additional code is generated:

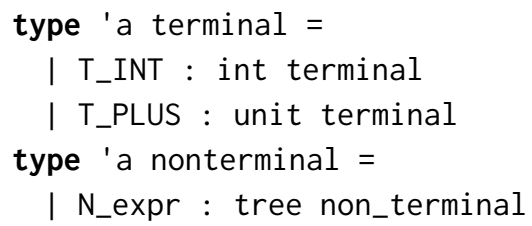




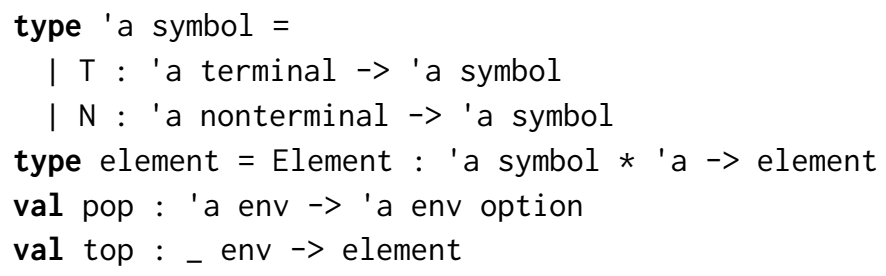

The 'a terminal and 'a nonterminal types contain one constructor per symbol, but they do not carry any data; they merely constrain their type parameter ' a to be equal to the type of the corresponding semantic value. The element type packs an 'a symbol together with the semantic value ' a, for an existential variable ' $a$ - this is a typical GADT encoding of a strong dependent pair, here $\Sigma(s:$ Symbol). SemType $(s)$. The function top provides access to the symbol and semantic value at the top of the stack, and pop removes one element off the stack.

Remark: The GADT proposed in Pottier and Régis-Gianas [2006] contain more fine-grained information than the types above. Not only do they allow to distinguish each symbol of the grammar, they include different constructors for each state in the parsing automaton, which keeps fine-grained typing information on the transitions from one state to another. In particular, it lets us statically predict, from the element on the top of the stack, which elements may be below it on the stack.

The simpler GADTs used by Merlin does not suffice to give a fully-typed presentation of the stack: ' a env remains an abstract type with more information, and top is a lossy projection. On the other hand, they are solely determined by the grammar structure (which the user wrote) rather than the parsing automaton (produced by the compiler), so they are much simpler to understand and use.

3.1.2 Integration and Other Uses. The work on an incremental Menhir interface started within Merlin in December 2013. It was adapted by François Pottier and merged in the upstream Menhir code in December 2014. Since its integration, three major use-cases were:

(1) Error message support. The complete API for the parser state exposes the automaton state number, which makes it easy to index an error message on the error state - an approach first proposed by Jeffery [2003]. More complete approaches are possible by inspecting the parsing stack; in particular, it enables fetching AST fragments around the error to print their location or pretty-print their source code in the error message. This is demonstrated in Pottier [2016], which builds on Menhir's incrementality support and typed stack API.

Other error-message strategies were implemented on top of the stack introspection APIs; we know of developments within Merlin itself, and in the frontend of the Reason language at Facebook.

(2) Generalized lexer hacks. Some languages ( $\mathrm{C}$ and Bash are notable example) are specified with a separate lexer and parser, but actually require a communication from the parser to the lexer, as the same source text may need to be recognized as different tokens depending on the current parsing state - this is called the "lexer hack", traditionally implemented through subtle use of mutable state. Using Menhir's incremental parsing API, one can pass the parsing state as a lexer parameter, and then use parsing stack inspection in the lexer as a pure approach to parser-lexer communication. This is used in the Bash parser of Jeannerod, Régis-Gianas, and Treinen [2017].

(3) Simulating local GLR parsing. GLR parsing can parse arbitrary context-free grammars by forking the parsing along different branches on ambiguities. The pure parsing interface allows to simulate this by doing the backtracking by hand, feeding the same parsing state to different 
continuations. This is less efficient than GLR algorithms that share more data between parsing threads, but it is very convenient to deal with local/bounded non-LR ambiguities. This is used in the Reason codebase, and also in Jeannerod, Régis-Gianas, and Treinen [2017].

\subsection{Partial Parsing With Error Recovery}

Recovery is the ability to handle partially incorrect files in Merlin, by asking the parsing and typechecking code to recover from unexpected / erroneous or unterminated input. Error resilient parsing is done by meta-programming the parser, using an extension to Menhir. On the type checking side, the changes are more mundane: the ability to recover from type errors in a few select points as well as a few heuristics to get a useful type derivation from the correct parts of the program.

From a software design point of view, recovery changes the possible outcome of the frontend from either a typed AST or an exception (representing a lexing/parsing/typing error) to a typed AST and a list of exceptions. This requires Merlin to produce an AST for any input and to type this AST, however wrong it is. In exchange, the rest of Merlin features always expect a typed AST to be available, although it may contain incorrect sub-derivations.

The parser enters a recovery state when receiving a token that is not expected in the current context. LR parsing guarantees that an error is detected as early as possible: the erroneous token is the first one for which there is no valid parse. The recovery algorithm makes use of this property by not trying to backtrack in general: all the input that has been parsed is kept - even though the most satisfying correction for the user could have been to drop a token. The recovery will only try to complete the parse by filling holes, either by synthesizing values or by resuming the parse using tokens coming later.

The details of how the Merlin authors guide OCaml parsing error recovery, described in the following sections, rely on both language-agnostic algorithms and heuristics that are guided by annotations on the language grammar. This approach should work in any language server, and in particular it is easy to add recovery capabilities to a parser, for any language, written using the Menhir parser generator.

In particular, the Merlin authors are adapting this work to improve the frontend of the Reason language - which is supported by Merlin - and studied the needs of the prototype Andromeda proof assistant being designed in Ljubljana - which hasn't adopted the Merlin tooling yet.

\subsection{Parsing Recovery: Synthesizing Values}

Consider the case where no more input is available (for instance, the user is typing a new file and only a prefix is available). The current parse represents an AST being filled from left to right, where all nodes on the right are missing at some point.

The authors of Merlin wrote a Menhir extension that automatically completes this partial AST, guided by the grammar and a few extra annotations. The technical details are out of the scope of this article, but the main idea is that, from a parser continuation, it is possible to synthesize a new symbol (terminal or non-terminal) to continue parsing, such that this repeated process always produces a complete AST after a finite number of steps.

In the general case, many different completions are possible, and some will produce completed parse trees that are more likely to make the user-written segments well-typed - and thus enable type-level feedback on user code - than others. To guide the recovery process, the Merlin authors annotated the input grammar to specify a "cost" for each shift/reduce transition happening during completion - transitions that have lower chances of producing a sensible program are given a higher cost. The completion algorithm then searches for a completion sequence of minimum combined cost. 
3.3.1 Recovery Annotations. To be able to produce an AST from a completed parse tree, each synthesized symbol must also come with a semantic value; this is also provided by annotations. For example, Merlin's OCaml grammar contains the following annotated definitions

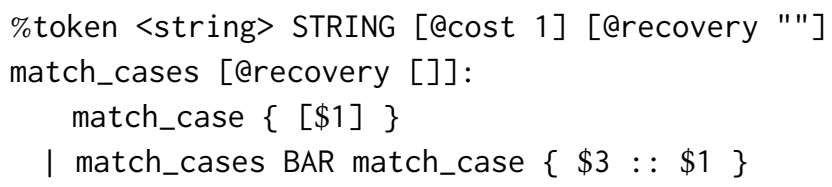

The annotation [@cost 1] gives a relatively low cost to synthesizing a missing STRING token, and [@recovery ""] suggests pretending that the empty string was parsed. The match_cases nonterminal is also provided with a semantic value to recover (the empty list of clauses). Synthesizing a complete non-terminal at once is much more robust than trying to complete with tokens only.

Semantic actions must be well-behaved for the recovery to work properly:

- Since the recovery can explore different branches and thus execute user code multiple times, actions should be observably pure.

- Errors should be logged and not affect the parser control flow. If an action can fail (raise an exception), it should be annotated with an infinite cost to avoid selecting it during recovery.

3.3.2 Integration and Adoption. The generic annotation mechanism, and the ability to postprocess them in tandem with a description of the generated parser automaton, were developed for Merlin's fork of Menhir over the course of 2015, and upstream in 2017. We are not currently aware of adoption by others than Merlin authors, but the Menhir designer, François Pottier, seems relieved that some of the features requested for Menhir can now be implemented as third-party annotation processors.

\subsection{Parsing Recovery: Reconnecting to User Input}

After encountering errors in the input file, the completion mechanism will synthesize symbols, but it would be nice to resume using the normal input as soon as possible. There is a tension between filling the AST, which will succeed but may drop the user input after the error, and resuming parsing, which is less reliable but will offer a better user experience if successful.

At any point, the recoverer has the choice between synthesizing one more value or consuming a token. Systematically trying both would lead to unacceptable performance. The main heuristic that drives Merlin's implementation is indentation, on the assumption that lexical indentation follows nesting of constructions in the grammar.

Precisely, Merlin compares the column of the current token and the column of the item at the top of the parser stack:

- If the parser is more indented, Merlin considers that the token should attach to a construction that is outside of the one the parser is currently parsing, so Merlin will do a synthesis step.

- If the parser is at a lesser or equal level of indentation, Merlin will try to consume the token, and if this fails, drop the token and fallback to synthesis.

While the indentation assumption is not true in practice for all indentation styles, this heuristic gives a satisfying behavior most of the time and should work for many languages.

\section{CONCLUSION}

\subsection{Future Work}

4.1.1 Memoized LR Reductions. It is possible to memoize the effect of parsing a sequence of tokens from a given LR automaton state, compressing a series of shift/reduce actions into a single 
effect on the parsing stack, the addition of a semantic value. Implementing this for Merlin is planned; it would allow a form of incrementality that can reuse work not only on valid (or recovered) prefixes on the input, but also share work across different parts of the buffer, and instantly re-parse input that was intermittently placed after an unrecoverable error.

4.1.2 Menhir Development Kit. Merlin changed Menhir to support adding arbitrary annotations to a grammar, and processing those annotations externally - a new Menhir mode provides access to serialized forms of both the annotated grammar and the compiled parsing automaton. This is growing into a more general "Menhir SDK" library, allowing various previously-unplanned uses of the grammar and automaton. For example, a work in progress is an algorithm to pretty-print parsing derivations on the concrete syntax, which is useful for teaching and grammar-engineering purposes.

Menhir supports parametric rules, abstracting some rules over a symbol or sequence of symbols, and inlining applications to specific parameters at compile-time. Their presence creates a distance between the user grammar and the grammar exposed by the introspection API, which is the postinlining grammar. There is work ongoing on "un-inlining" approaches to recover applications of parametrized rules, but higher-order parameters make this challenging or impossible in the general case.

\subsection{Related Work}

4.2.1 Tim Wagner's Work on Incremental Parsing. An interesting body of work on incremental parsing is Tim Wagner's PhD thesis work in the 1990s [Wagner 1998; Wagner and Graham 1997, 1998]. The Merlin authors were not aware of this work as they designed their incremental frontend, so it is interesting to compare their approaches.

Wagner's work studies lexer and parser generators as used in imperative and object-oriented programming languages; general differences with the Merlin work arise from this difference in paradigm. A lexer or parser generator for OCaml will typically generate a nest of tail-recursive functions; on the other hand, a C implementation would typically work with a single execution loop, mutating a bit of state that represents the control (the next automaton state to consider). In other words, lexers and parsers in imperative languages tend to have their control flow reified in data and define their semantic actions as manipulating mutable values. Lexers and parser in functional language tend to have an abstract control flow, but return immutable values. Incremental lexing and parsing requires the best of both world: reified control flow, and pure actions returning immutable values. Wagner had to take care of isolating actions and preserving meaningful notions of physical identity to rely on; Merlin can just compare pure values, but had to add continuation-passing or control-inversion mechanisms.

Wager also points out that, for proper incrementality support, lookahead must be carefully handled: a lexer or parser decision may depend on what follows next in the buffer, and be invalidated by buffer changes. LR parsers typically use a single lookahead token, but lexers may use arbitrary lookahead; Wagner keeps track of the dynamic lookahead for incremental lexing. This is not implemented in Merlin, as the OCaml lexer does not require arbitrary lookahead, but it may be important for adaptation to other languages.

Finally, Wagner established some complexity results, characterizing notions of optimal algorithms for incremental processing. In particular, parsing spines may sometimes have to be rebalanced to preserve logarithmic access costs and avoid linear worst-cases. These results typically do not include the cost of producing the AST structure, or require changes to this structure - rebalancing a partial list as a tree for logarithmic access is pointless if the semantic action then immediately builds a linear list to represent this AST. 
An implementation inspired by Tim Wagner's work is the Tree-Sitter project developed by Max Brunsfeld at Github. One important difference is that Tree-Sitter uses a GLR parser algorithm. This project seems focused on providing reasonable (but possibly approximate) parsers for a large number of languages, for integration within the Atom code editor.

Tree-sitter also implements a "contextual scanner", a lexer that is indexed over the parser state. This has been done manually in some OCaml projects, but it could be added to Menhir as a general feature.

Another, closer implementation of Tim Wagner's ideas can be found in the work on the Eco editor that we discuss later in this section. The Eco project did not implement AST rebalancing, and the authors report that this does not seem to create performance issues in practice.

4.2.2 Yacc Error Recovery. It is interesting to compare Merlin's approach to syntax error recovery with Yacc's error recovery using the ERROR tokens. Yacc lets users integrate error tokens in any rule, to print a user-written error message or to provide a default action for recovery. If a parsing error is encountered, it will typically not be exactly in the place of an error token in an error-handling rule, so Yacc has an algorithm to perform "error reductions" until it finds an enclosing error-handling rule.

Starting from the same state, the strategies can be summarized as "Yacc drops information that separates it from an error handling rule", while "Merlin invents information that separates it from a possible recovery".

The benefits of Merlin's strategy are significant for interactive use: while inventing information could mean generating garbage, the process can actually be guided by the user. Preserving information means that the last tokens typed by the user are considered during parsing and further analyses.

For instance, in let $f x$ : int $=$, the prefix is correct: Merlin knows the context should be completed by an expression of type int and that a variable $x$ is in scope. With Yacc, this property only holds if there is an error-handling rule covering exactly this case. It is difficult to add enough error-handling rules: there will need to be many, and they should not conflict.

Merlin is also more flexible in the handling of ambiguities: while Yacc rejects ambiguous error rules (causing shift/reduce or reduce/reduce conflicts), Merlin allows ambiguities and uses its cost model for token synthesis to direct the non-deterministic search.

4.2.3 Eco. The Eco editor [Diekmann and Tratt 2014] supports language composition (mixing of programming languages within the same editor file), and relies on incremental parsing to provide syntax-aware editing. Eco bridges the gap between syntax-directed editing and textual editing by using as its main source representation a concrete syntax tree (incrementally constructed) structured to retain all textual information, in particular whitespace and comments.

Merlin uses a less principled approach, working with parse trees and ASTs, and relying on source positions stored in the AST to map AST nodes to source text fragments.

4.2.4 Editor Modes. Many IDEs provide language-aware support, generally for mainstream languages with a large user base: $\mathrm{C} / \mathrm{C}++$, Java, etc. An early example is the Delphi IDE for Pascal. Those systems offer a very fine-grained integration of the language - Delphi directly hooks into its own Pascal compiler. For people who are not programming language researchers, an IDE may be their main way to familiarize themselves with details of the language features (scope resolution, type inference...).

On the other hand, language-aware support for functional languages with a rich type system has a more checkered history. For example, the Scala community went through many iterations of 
its incremental type-checker. IntelliJ for Scala, for example, would use a type checker with slightly different behavior from the Scala compiler, resulting in inconsistencies that users would find jarring.

An interesting design space is the editor modes for proof assistants, that brought several innovations such as typed holes. In a proof assistant, the editor experience is central, as proofs are rarely run; in some sense, the frontend provides the user experience.

\section{ACKNOWLEDGMENTS}

The main implementors of Merlin are Frédéric Bour and Thomas Refis, two vim users - with financial support from Jane Street. Simon Castellan added support for Emacs, Luc Rocher for Sublime Text, Hackwaly for Visual Studio Code, Facebook for Atom, Raphaël Proust for Acme. Darrin Morrison implemented the Language Server Protocol support, David Allsopp ported the tool on Windows, and Gemma Gordon helped with project management.

The mechanisms for parser-agnostic syntactic recovery was designed and implemented in Menhir in collaboration with Philipp Haselwarter, during a Ljubljana visit funded by Andrej Bauer.

We also wish to thanks the anonymous reviewers, and Lukas Diekmann, for their detailed comments.

\section{REFERENCES}

Lukas Diekmann and Laurence Tratt. 2014. Eco: A language composition editor. In Software Language Engineering (SLE).

Matthew Flatt, Eli Barzilay, and Robert Bruce Findler. 2009. Scribble: closing the book on ad hoc documentation tools. In ICFP.

Nicolas Jeannerod, Yann Régis-Gianas, and Ralf Treinen. 2017. Having Fun With 31.521 Shell Scripts. (April 2017). https://hal.archives-ouvertes.fr/hal-01513750.

Clinton L. Jeffery. 2003. Generating LR Syntax Error Messages from Examples. TOPLAS 25, 5 (Sept. 2003), 631-640.

Cyrus Omar, Ian Voysey, Michael Hilton, Joshua Sunshine, Claire Le Goues, Jonathan Aldrich, and Matthew A. Hammer. 2017. Toward Semantic Foundations for Program Editors. In SNAPL.

François Pottier. 2016. Reachability and error diagnosis in LR(1) parsers. In Proceedings of the 25th International Conference on Compiler Construction (CC 2016). 88-98.

François Pottier and Yann Régis-Gianas. 2006. Towards efficient, typed LR parsers. In ACM Workshop on ML (Electronic Notes in Theoretical Computer Science), Vol. 148. 155-180.

Tim A. Wagner. 1998. Practical Algorithms for Incremental Software Development Environments. Ph.D. Dissertation. Berkeley. Tim A. Wagner and Susan L. Graham. 1997. History-Sensitive Error Recover. IEEE Trans. on Software Engineering (1997).

Tim A. Wagner and Susan L. Graham. 1998. Efficient and Flexible Incremental Parsing. ACM Trans. on Programming Languages and Systems 20, 5 (1998). 